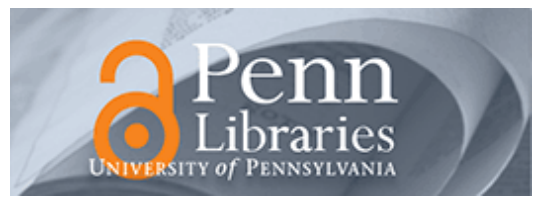

University of Pennsylvania

ScholarlyCommons

Statistics Papers

Wharton Faculty Research

$5-15-2002$

\title{
Bayesian Nonparametric Point Estimation Under a Conjugate
} Prior

Xuefeng Li

University of Pennsylvania

Linda H. Zhao

University of Pennsylvania

Follow this and additional works at: https://repository.upenn.edu/statistics_papers

Part of the Statistics and Probability Commons

\section{Recommended Citation}

Li, X., \& Zhao, L. H. (2002). Bayesian Nonparametric Point Estimation Under a Conjugate Prior. Statistics \& Probability Letters, 58 (1), 23-30. http://dx.doi.org/10.1016/S0167-7152(02)00097-4

This paper is posted at ScholarlyCommons. https://repository.upenn.edu/statistics_papers/570

For more information, please contact repository@pobox.upenn.edu. 


\title{
Bayesian Nonparametric Point Estimation Under a Conjugate Prior
}

\author{
Abstract \\ Estimation of a nonparametric regression function at a point is considered. The function is assumed to lie \\ in a Sobolev space, $S_{q}$, of order $q$. The asymptotic squared-error performance of Bayes estimators \\ corresponding to Gaussian priors is investigated as the sample size, $n$, increases. It is shown that for any \\ such fixed prior on $S_{q}$ the Bayes procedures do not attain the optimal minimax rate over balls in $S_{q}$. This \\ result complements that in Zhao (Ann. Statist. 28 (2000) 532) for estimating the entire regression \\ function, but the proof is rather different.

\section{Keywords} \\ nonparametric regression, point estimation, Bayesian procedure, Gaussian prior, optimum rate \\ Disciplines \\ Statistics and Probability
}




\title{
Bayesian nonparametric point estimation under a conjugate prior
}

\author{
Xuefeng Li \\ Linda H. Zhao * \\ Statistics Department \\ University of Pennsylvania
}

\begin{abstract}
Estimation of a non-parametric regression function at a point is considered. The function is assumed to lie in a Sobolev space, $S_{q}$, of order $q$. The asymptotic squared-error performance of Bayes estimators corresponding to Gaussian priors is investigated as the sample size, $n$, increases. It is shown that for any such fixed prior on $S_{q}$ the Bayes procedures do not attain the optimal minimax rate over balls in $S_{q}$. This result complements that in Zhao (2000) for estimating the entire regression function, but the proof is rather different.
\end{abstract}

Key words: nonparametric regression, point estimation, Bayesian procedure, Gaussian prior, optimal rate.

* Research supported in part by NSF grant DMS-9971848. Linda Zhao, 3000 SH-DH, 3620 Locust Walk, Philadelphia, PA 19104-6302 


\section{Introduction}

Within the past two decades nonparametric regression has become an important, widely used statistical methodology. More recently there has been increasing interest in the possibility of effectively using a Bayesian approach for such situations. This paper involves one step in that direction.

We investigate an aspect of the performance of the Bayes estimator for a natural conjugate prior. (These priors correspond to infinite dimensional Gaussian distribution.) Of interest is the asymptotic performance of the estimator of the regression function, $f$, at a given point, $x_{0}$.

As is customary, we assume that regression function lies in a standard function space - in this case a Sobolev space of specified smoothness. Consistent with this we derive the prior to be supported on this Sobolev space.

We show that for any such prior the Bayes estimators for samples size $n$ do not attain the optimal minimax rate of squared error risk.

Zhao (2000) demonstrates an analogous deficiency of Bayes procedures for the problems of estimating the entire regression function. For that problem she also constructs a nonconjugate prior distribution whose Bayes estimators do attain the optimal minimax rate. It follows, however, from Cai, Low and Zhao (2001) that these estimators will not attain the optimal minimax rate for estimating $f\left(x_{0}\right)$. The question is thus an open on of whether there exists a prior on the Sobolev space whose Bayes procedures attain this rate for estimating $f\left(x_{0}\right)$. We suspect such prior exist but have not (yet) succeeded in constructing them.

For further background on problems of this nature and additional references we refer the

reader to Zhao (2000). We close the introduction by noting two additional features of the results in the present manuscript.

First, we prove here an additional result that shows there do exist Gaussian priors supported outside the given Sobolev-space whose estimators of $f\left(x_{0}\right)$ do attain the optimal minimax rate. This result is analogous to one in Zhao (2000) for estimating all of $f$; however the appropriate priors are different in the two problems. (This is consistent with the fact that the minimax rates are also different.) Second, although the main theorem here has 
analogies to the main result in Zhao (2000), the proof is rather different.

\section{Preliminaries}

In a standard nonparametric regression problem one observes $\left(x_{i}, z_{i}\right), i=1, \ldots, n$ where

$$
z_{i}=f\left(x_{i}\right)+\epsilon_{i}, \quad i=1, \ldots, n .
$$

Here we take $x_{i}=i /(n+1)$ to be equally spaced on $[0,1]$ and we take $\epsilon_{i} \stackrel{i . i . d .}{\sim} N\left(0, \sigma^{2}\right)$. For simplicity assume $\sigma^{2}=1$. Our goal is to estimate $f\left(x_{0}\right)$, the value of $f$ at a given point, $x_{0} \in[0,1]$. The loss function is squared error loss:

$$
L\left(\hat{f}\left(x_{0}\right), f\left(x_{0}\right)\right)=\left(\hat{f}\left(x_{0}\right)-f\left(x_{0}\right)\right)^{2} .
$$

Donoho, Liu and MacGibbon (1990), Brown and Low (1996) and Brown and Zhao (2001) show the following equivalence results. Suppose $f$ is expressed by an orthonormal basis $\left\{\varphi_{i}(x)\right\}$ on $\mathscr{L}_{2}=\left\{f: \int_{0}^{1} f^{2}(x) d x<\infty\right\}$, i.e.

$$
f(x)=\sum \theta_{i} \varphi_{i}(x) .
$$

Then we can construct $\left\{y_{i}\right\}$ as a (randomized) function of $\left\{z_{i}\right\}$ such that

$$
y_{i}=\theta_{i}+\frac{1}{\sqrt{n}} \epsilon_{i}, i=1, \ldots, \epsilon_{i} \stackrel{i . i . d .}{\sim} N(0,1) .
$$

Estimating a functional such as $f\left(x_{0}\right)$ is asymptotically equivalents to estimating the matching functional of $\theta=\left\{\theta_{i}\right\}$. (Brown and Zhao (2001) give an equivalence construction that is also valid if the $x_{i}$ are themselves observations of $i . i . d$. random variables on $[0,1]$.)

To be explicit we take $\varphi_{i}$ to be the usual Fourier basis on $[0,1]$. Thus, for $x \in[0,1]$

$$
\begin{gathered}
\varphi_{0}(x)=1 \\
\varphi_{2 k-1}(x)=2^{-1 / 2} \cos (2 \pi k x) \\
\varphi_{2 k}(x)=2^{-1 / 2} \sin (2 \pi k x), \quad k=1,2, \ldots .
\end{gathered}
$$

Then $\theta_{i}=\int_{0}^{1} f(x) \varphi_{i}(x) d x, i=0, \ldots$ If $\hat{\theta}=\left\{\hat{\theta}_{i}\right\}$ is an estimator of $\theta=\left\{\theta_{i}\right\}$ then

$$
\hat{f}\left(x_{0}\right)=\sum a_{i} \hat{\theta}_{i}, \quad a_{i}=\varphi_{i}\left(x_{0}\right)
$$


is the matching estimator of $f\left(x_{0}\right)$. This can conveniently be rewritten as $\hat{f}\left(x_{0}\right)=a^{\prime} \hat{\theta}$ where $a=\left\{a_{i}\right\}, \hat{\theta}=\left\{\hat{\theta}_{i}\right\}$. If $T$ denotes an estimator of $f\left(x_{0}\right)$ then the risk function is of course

$$
R\left(T, f\left(x_{0}\right)\right)=E\left(T-f\left(x_{0}\right)\right)^{2} .
$$

When $f$ is assumed to be in a Sobolev ball $S_{q}(B)=\left\{\left\{\theta_{i}\right\}: \sum i^{2 q} \theta_{i}^{2} \leqslant B\right\}$ when $q>1 / 2$ the optimal minimax rate in the present problem is known to be $n^{-(2 q-1) / 2 q}$, i.e.,

$$
0<\inf _{T} \sup _{\theta \in S_{q}(B)} n^{\frac{2 q-1}{2 q}} R\left(T, f\left(x_{0}\right)\right)<\infty .
$$

This rate was established by Wahba (1975); see also Donoho and Low (1992). We assume throughout that $q>1 / 2$.

\section{Main results}

How does a Bayesian estimator perform for this nonparametric point estimation problem? We are especially interested in the question of whether the Bayes solution resulting from a Gaussian prior possesses the optimal property defined in (4).

Zhao (2000) dealt with Bayesian estimation of the entire function. We establish similar results for estimating $f\left(x_{0}\right)$ under square error loss. The general results have points of similarity, but there are also some differences and the methods of proof are rather different.

A product Gaussian prior

$$
\pi(\theta)=\prod N\left(0, \tau_{i}^{2}\right)
$$

has support on $S_{q} \triangleq\left\{\left\{\theta_{i}\right\}: \sum i^{2 q} \theta_{i}^{2}<\infty\right\}$ if and only if

$$
\sum_{i} i^{2 q} \tau_{i}^{2}<\infty
$$

It is straightforward to compute the posterior mean of the prior to be

$$
\hat{\theta}_{i}=\frac{\tau_{i}^{2}}{\tau_{i}^{2}+\frac{1}{n}} y_{i} .
$$

For details of both assertion see Zhao (2000). The Bayes estimator of $f\left(x_{0}\right)$ is then easily calculated to be

$$
\hat{T}=\sum a_{i} \hat{\theta}_{i}=a^{\prime} \hat{\theta} .
$$


We first show that there exist independent normal priors whose Bayes procedure attains the optimal minimax rate, but these priors are not supported on $S_{q}$.

Theorem 3.1 Let $\tau_{i}^{2}=i^{-2 p}$ in (5). When $p>\max \left(\frac{q}{2}, \frac{1}{2}\right)$ the Bayes estimator $\hat{T}$ in (7) has the minimax rate $n^{-m(p, q)}$, where $m(p, q)=\min \left(1-\frac{1}{2 p}, \frac{2 q-1}{2 p}\right)$. To be more precise,

$$
0<\lim _{n \rightarrow \infty} \sup _{\theta \in S_{q}(B)} n^{m(p, q)} R\left(a^{\prime} \hat{\theta}, f\left(x_{0}\right)\right)<\infty .
$$

In particular, the Bayes estimator attains the optimal minimax rate if and only if $p=q$.

Proof: Take $B=1$ with no loss of generality. The risk function can be written as

$$
R\left(a^{\prime} \hat{\theta}, a^{\prime} \theta\right)=\operatorname{Var}\left(a^{\prime} \hat{\theta}\right)+\operatorname{Bias}^{2}\left(a^{\prime} \hat{\theta}, a^{\prime} \theta\right) .
$$

Note that if $b_{i}>0$, then

$$
\sup _{\sum b_{i} \theta_{i}^{2} \leqslant 1}\left(\sum w_{i} \theta_{i}\right)^{2}=\sum \frac{w_{i}^{2}}{b_{i}} .
$$

Then, for the squared bias in (8)

$$
\begin{aligned}
\sup _{\sum i^{2 q} \theta_{i}^{2} \leqslant 1} \operatorname{Bias}^{2}\left(a^{\prime} \hat{\theta}, a^{\prime} \theta\right) & =\sup _{\sum i^{2 q} \theta_{i}^{2} \leqslant 1}\left[\sum a_{i}\left(\frac{\tau_{i}^{2}}{\tau_{i}^{2}+1 / n} \theta_{i}-\theta_{i}\right)\right]^{2} \\
& =\sup _{\sum i^{2 q} \theta_{i}^{2} \leqslant 1}\left[\sum a_{i} \frac{\theta_{i}}{1+n \tau_{i}^{2}}\right]^{2} \\
& =\sum a_{i}^{2} \frac{\left(1+n i^{-2 p}\right)^{-2}}{i^{2 q}} \\
& \sim n^{\frac{1-2 q}{2 p}}
\end{aligned}
$$

where the last assertion comes from

$$
\sum \frac{\left(1+n i^{-2 p}\right)^{-2}}{i^{2 q}} \sim n^{\frac{1-2 q}{2 p}}
$$

and from

$$
a_{2 k-1}^{2}+a_{2 k}^{2}=1, \forall k \geqslant 1
$$

For the variance term

$$
\operatorname{Var}\left(a^{\prime} \hat{\theta}\right)=\frac{1}{n} \sum a_{i}^{2}\left(\frac{i^{-2 p}}{i^{-2 p}+\frac{1}{n}}\right) \sim n^{-\left(1-\frac{1}{2 p}\right)}
$$


where we have again used (12).

Hence the minimax rate for $\mathbf{a}^{\prime} \hat{\theta}$ is $n^{-\min (1-1 /(2 p),(2 q-1) / 2 p)}$. And when $p=q, \min (1-$ $1 /(2 p),(2 q-1) / 2 p)$ achieves its maximum value $\frac{2 q-1}{2 q}$, for which the corresponding rate is just the optimal rate.

Remark: Among priors with $\tau_{i}^{2}=i^{-2 p}$ the Bayes estimator is optimal only when $p=q$. But in that case both the prior and the posterior distribution have measure 0 on the space $S_{q}$ of the interest.

The next theorem builds from Theorem 3.1 and gives a more general result about Bayesian approaches.

Theorem 3.2 There does not exist a Gaussian prior on $S_{q}$ such that the corresponding sequence of Bayes procedures attains the optimal minimax rate. That is, if $\Sigma$ is the covariance matrix of a Gaussian measure on $S_{q}$, then the Bayes estimator $\hat{T}$ of $f\left(x_{0}\right)=a^{\prime} \theta$ must have

$$
\lim _{n \rightarrow \infty} \sup _{\theta \in S_{q}(B)} n^{\frac{2 q-1}{2 q}} R\left(\hat{T}, a^{\prime} \theta\right)=\infty .
$$

Before we prove the above theorem let us derive some basic facts as lemmas.

Lemma 3.1 Let $D, W$ be positive definite $m \times m$ matrices and $b$ an $m$ dimensional vector. Then

$$
\sup _{\xi^{\prime} D \xi \leqslant 1}\left(b^{\prime} W \xi\right)^{2}=b^{\prime} W D^{-1} W b
$$

Proof: This standard result is the matrix generalization of (9). We omit the proof.

Lemma 3.2 Let $\mathscr{P}_{m}=\{P: P$ is an $m \times m$ positive definite matrix with maximum eigenvalue $<1\}$ and $\vec{u}$ be a unit vector. Then

$$
\inf _{P \in \mathscr{P}_{m}}\left\{\vec{u}^{\prime} P^{2} \vec{u}+\operatorname{Tr}\left(P^{-1}-I\right)\right\}>0.889 .
$$

Proof: Write $P=O \Lambda O^{\prime}$, with $O$ some orthonormal matrix and $\Lambda=\operatorname{Diag}\left(\lambda_{i}\right)$. Here $\left\{\lambda_{i}\right\}$ are the eigenvalues of $P$. Let $\vec{v}=O^{\prime} \vec{u}$. Then $\vec{v}$ is also a unit vector, i.e. $\sum v_{i}^{2}=1$. Then,

$$
\begin{aligned}
& \vec{u}^{\prime} P^{2} \vec{u}+\operatorname{Tr}\left(P^{-1}-I\right) \\
= & \vec{v}^{\prime} \Lambda^{2} \vec{v}+\sum \frac{1}{\lambda_{i}}-m \\
= & \sum \lambda_{i}{ }^{2} v_{i}{ }^{2}+\sum \frac{1}{\lambda_{i}}-m .
\end{aligned}
$$


Hence

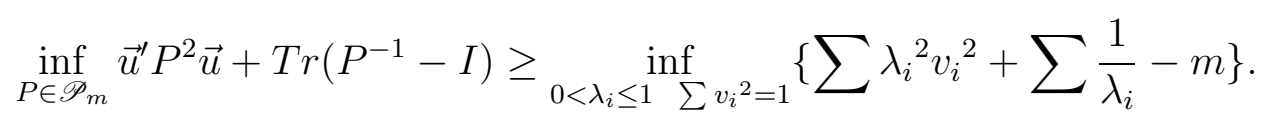

If $1 / 2<v^{2} \leq 1$ the function $\lambda^{2} v^{2}+1 / \lambda$ attains its minimum over $0 \leq \lambda \leq 1$ at $\lambda=1 /\left(2 v^{2}\right)^{1 / 3}$. If $0 \leq v^{2} \leq 1 / 2$ then this function attains its minimum on this region at $\lambda=1$. At most one $v_{j}^{2}$ can satisfy $v_{j}^{2}>1 / 2$ since $\sum v_{j}^{2}=1$. Suppose there is one such $v_{j}$. Then

$$
\begin{aligned}
& \inf _{0<\lambda_{i} \leq 1} \sum v_{i}^{2}=1 \\
\geq & \left.\inf _{1 / 2 \leq v_{j}^{2} \leq 1}\left(2^{-2 / 3}+2^{1 / 3}\right) v_{j}{ }^{2} v_{i}{ }^{2}+\sum \frac{1}{\lambda_{i}}-m\right\} \\
\geq & \inf _{1 / 2 \leq v_{j}^{2} \leq 1}\left(2^{-2 / 3}+2^{1 / 3}\right) v_{j}{ }^{2 / 3}-v_{j}{ }^{2} \\
= & .88988 .
\end{aligned}
$$

On the other hand, if all $v_{j}^{2} \leq 1 / 2$ then

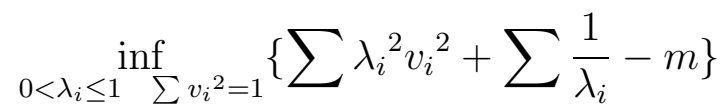

$$
\begin{aligned}
& \geq \sum v_{j}^{2}+m-m=1 \text {. }
\end{aligned}
$$

Proof: 1. It suffices to consider prior having mean 0. Since $f\left(x_{0}\right)=a^{\prime} \theta$ the Bayes estimator will be $\hat{T}=a^{\prime} \hat{\theta}$ with

$$
\hat{\theta}=a^{\prime} \sum\left(\frac{I}{n}+\Sigma\right)^{-1} Y
$$

Now,

$$
\begin{aligned}
R\left(\hat{T}, a^{\prime} \theta\right) & \geqslant \operatorname{Bias}^{2}\left(\hat{T}, a^{\prime} \theta\right) \\
& =\left[a^{\prime}\left(\sum\left(\frac{I}{n}+\Sigma\right)^{-1}-I\right) \theta\right]^{2} \\
& =\left(a^{\prime}(I+n \Sigma)^{-1} \theta\right)^{2} \\
& =\left(a^{\prime} V \theta\right)^{2}
\end{aligned}
$$

with $V$ defined as

$$
V=(I+n \Sigma)^{-1}
$$

Notice that all eigenvalues of $V$ are between 0 and 1 and

$$
\Sigma=n^{-1}\left(V^{-1}-I\right) .
$$


Given an integer $m$ let $D$ denote the $(m \times m)$ diagonal matrix with diagonal entries $(m+i)^{2 q}, i=1, \ldots, m$. Let $W$ denote the $(m \times m)$ matrix composed of the $(m+1)^{t h}, \ldots .2 m^{t h}$ rows and columns of $V$ and let $b$ consist of the corresponding coordinates of $a$. Note that $D, W, b$ all depend on $m$, but the dependence is suppressed in the notation. From (16) and $(17)$,

$$
\begin{aligned}
& \sup _{\sum i^{2 q} \theta_{i}^{2} \leqslant 1} \operatorname{Bias}^{2}\left(\hat{T}, a^{\prime} \theta\right) \geqslant \sup _{\sum_{i=m+1}^{2 m} i^{2 q} \theta_{i}^{2} \leqslant 1: \theta_{i}=0} \text { if } i \notin[m+1,2 m] \\
&=\operatorname{sias}^{2}\left(\hat{T}, a^{\prime} \theta\right) \\
& \sup _{\xi^{\prime} D \xi \leqslant 1}\left(b^{\prime} W \xi\right)^{2}
\end{aligned}
$$

where $\xi$ corresponds to the vector in the previous expression having coordinates $\left(\theta_{m+1}, \ldots, \theta_{2 m}\right)$.

Hence, by Lemma 3.1

$$
R\left(\hat{T}, a^{\prime} \theta\right) \geqslant b^{\prime} W D^{-1} W b .
$$

2. Recall that $a_{2 k-1}^{2}+a_{2 k}^{2}=1, k=1, \ldots$. Hence in (20) $\|b\| \sim m / 2$. More precisely, for all $m>100$

$$
\|b\|^{2} \geqslant .49 m .
$$

Suppose that the assertion of the theorem is false. Then by (20) there is a $c<\infty$ such that

$$
n^{\frac{2 q-1}{2 q}} b^{\prime} W D^{-1} W b \leqslant c .
$$

Note that $D^{-1}-(2 m)^{-2 q} I$ is positive semi-definite. Hence (21) and (22) imply

$$
n^{\frac{2 q-1}{2 q}}(.49 m) \frac{1}{(2 m)^{2 q}} \vec{u}^{\prime} W^{2} \vec{u} \leqslant c
$$

where $\vec{u}=b /\|b\|$ is a unit vector.

Now take

$$
m=\left[n^{\frac{1}{2 q}}\left(\frac{2^{2 q}}{.4 \times .49} c\right)^{\frac{1}{1-2 q}}\right] .
$$

Then from (23)

$$
\vec{u}^{\prime} W^{2} \vec{u} \leqslant .4
$$

3. Now, for $m$ as in (24)

$$
\sum_{i=m+1}^{2 m} i^{2 q} \sum_{i i}=\frac{1}{n} \sum_{i=m+1}^{2 m} i^{2 q}\left(\left(V^{-1}\right)_{i i}-1\right)
$$




$$
\begin{aligned}
& \geqslant \frac{1}{n} m^{2 q} \sum_{i=m+1}^{2 m}\left(\left(V^{-1}\right)_{i i}-1\right) \\
& \geqslant c_{1} \operatorname{Tr}\left(W^{-1}-I_{m}\right) \quad \text { for }\left(V^{-1}\right)_{\text {diag }}>\left(V_{\text {diag }}\right)^{-1}
\end{aligned}
$$

with $c_{1}>0$, independent of $m$. Apply Lemma 3.2 and (25) to get

$$
\operatorname{Tr}\left(W^{-1}-I_{m}\right) \geqslant .48
$$

Hence

$$
\sum_{i=m+1}^{2 m} i^{2 q} \Sigma_{i i} \geqslant .48 c_{1} .
$$

As $n \rightarrow \infty$ there is an infinite sequence of arbitrarily large corresponding values of $m$ given by (24). Thus (27) yields

$$
\sum_{i=1}^{\infty} i^{2 q} \Sigma_{i i}=\infty .
$$

This establishes that the Gaussian prior is not supported on $S_{q}$.

Remark: Note that the preceding proof establishes a slightly stronger fact than claimed in (13). Namely, for any Gaussian prior on $S_{q}$ the squared bias does not converge at the optimal rate.

Remark: The preceding results explicitly concern one special formulation of $S_{q}$. However the same general results can be shown to apply to the more general problem of estimating $f$ at a point with parameter space $\left\{f=\sum \theta_{i} \phi_{i}(x): \quad \sum c_{i} \theta_{i}^{2}<\infty\right\}$, where $c_{i} \sim c i^{2 q}$. Only minor modifications of the preceding proof are needed.

\section{References}

[1] Brown, L.D. and Low, M.(1996). Asymptotic equivalence of nonparametric regression and white noise. Ann. Statist. 24 2384-2398

[2] Brown, L.D. and Zhao, L. (2001). Direct asymptotic equivalence of nonparametric regression and the infinite dimensional location problem. Technical report. (available from www-stat.wharton.upenn.edu/ ${ }^{\sim}$ lzhao). 
[3] Cai, T.T., Low, M.G. and Zhao, L. (2001) Tradeoffs between global and local risks in nonparametric function estimation. Technical report. (available from www-stat.wharton.upenn.edu/ ${ }^{\sim}$ lzhao).

[4] Donoho, D.L., Liu, R. and MacGibbon, B. (1990) Minimax risk over hyperrectangles and implications, Ann. Statist., 18, 1416-1437

[5] Donoho, D.L., Low, M.G. (1992) Renormalization exponents and optimal pointwise rates of convergence, Ann. Statist., 20, 944-970

[6] Wahba, G. (1975) Smoothing noisy data by spline functions, Numer. Math., v $24,383-393$

[7] Zhao, L. (2000) Bayesian aspects of some nonparametric problems. Ann. Statist., 28, 532-552 